Methods DM patients with daytime sleepiness or symptoms of respiratory failure were referred to the Regional Sleep Centre. They were admitted overnight for sleep study and respiratory assessment. If there was central hypoventilation causing respiratory failure, or obstructive sleep apnoea (OSA), they were offered non-invasive ventilation (NIV) or continuous positive airway pressure (CPAP) respectively. People without sleep disordered breathing with daytime sleepiness were assessed for modafinil, an alerting drug.

Results From May 2011 to May 2015, 120 people with DM had investigations. Mean age was 47 (SD 13, range 18-74), mean BMI $28 \mathrm{~kg} / \mathrm{m}^{2}(7,16-53)$ and mean Epworth Sleepiness Score 13 (5, 2-24). Mean Muscular Impairment Rating Scale was $3.85(0.7,2-5)$.

Mean FEV1 was $70 \%$ predicted (SD 22), FVC 68\% predicted (27), with a $>15 \%$ supine fall in FVC in $18 \%$. PI max, PE max or SNIP were below $50 \%$ predicted in $73 \%$ of people.

In this cohort, 32 people $(27 \%)$ had raised $\mathrm{CO}_{2}>6.2$ $\mathrm{mmHg}$; NIV was trialled and provided at home in 29 (90\% of those in whom it was indicated). Twelve people (37\%) have continued this, 17 (53\%) have returned it.

Twenty one people (18\%) were found to have OSA and 19 commenced CPAP (90\% of those in whom it was indicated). This was continued by seven people (33\%) and returned by 12 (57\%).

Thirty seven people (31\%) found to have no evidence of significant sleep-disorderd breathing were assessed for modafinil. Twenty seven people ( $73 \%$ of those in whom this was indicated) attended clinic for this: 16 (43\%) had good effect from modafinil and continue on it, $8(22 \%)$ had no effect or intolerance; in three $(8 \%)$ it was not prescribed.

Thirty people (25\% of total cohort) had no sleep disordered breathing and required no further input.

Conclusions DM is a heterogeneous disorder, with varying BMI, sleepiness, muscular and respiratory impairment. Overall, only $35 \%$ of the total cohort gained benefit from CPAP, NIV or modafinil and continued with this therapy.

\section{P116 IMPACT OF BARIATRIC SURGERY ON OSAS: A 4-YEAR EXPERIENCE}

V Palissery, S Kumar, D Ghosh, M O'Kane, MW Elliott. Leeds Teaching Hospitals NHS Trust, Leeds, UK

10.1136/thoraxjnl-2015-207770.253

Background Obstructive Sleep Apnoea Syndrome (OSAS) is common in morbidly obese patients. Previous studies indicate bariatric surgery reduces the severity of OSAS but may not cure it. We explored the impact bariatric surgery on patients who were commenced on CPAP prior to surgery.

Methods All morbidly obese patients who underwent bariatric surgery at our institution, between June 2010 and May 2014 who underwent sleep study (SS) or oximetry prior to bariatric surgery were included. The primary end point was cure (oximetry off CPAP showing either ODI less than 5 or ODI 5-15 with ESS $<10$ and no other symptoms of SDB) from OSAS. Secondary end points were weight loss achieved, improvement in OSAS and improvement in ESS. All data were obtained from electronic bariatric surgery and sleep service databases.

Results 184 patients underwent bariatric surgery. 97 (52.7\%) had SS or oximetry prior to surgery. (Figure 1) Mean ODI was 25 (95\% CI 19-30) and ESS 12 (95\% CI 10-13). Out of 46 patients considered for CPAP, 45 continued using CPAP perioperatively, one discontinued after failed trial. 20 (43.4\%) patients were considered cured from OSAS by 12-24 months. 17 (36.9\%) patients became asymptomatic and returned CPAP were considered to be cured clinically but not had SS post surgery. At 12 months post bariatric surgery, there were significant $(\mathrm{P}<0.0001)$ reductions in various parameters; means of difference in ODI 34, ESS 10 and BMI 17.8.

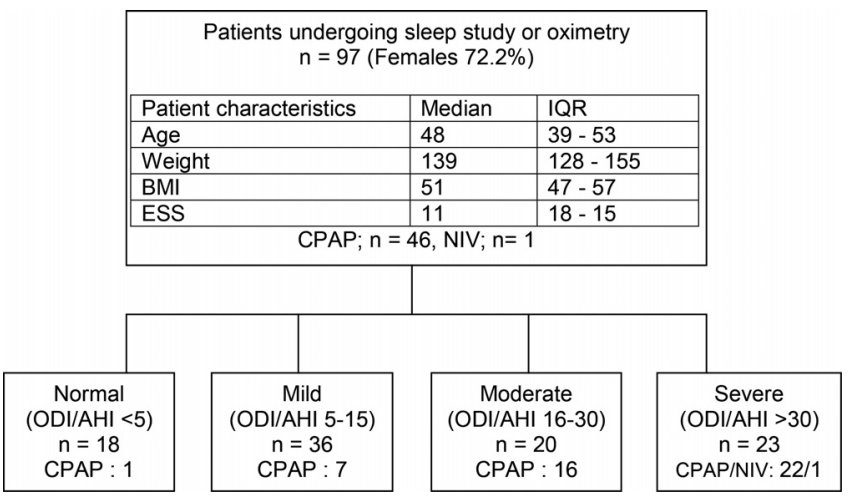

Abstract P116 Figure 1 Patient characteristics. ESS = Epworth Sleepiness Score, ODI = Oxygen Desaturation Index, AHI = Apnoea Hypopnoea Index

Discussion and conclusion In contrast to the meta-analysis (Greenburg et al. 2009) in which 62\% had residual disease in our cohort $80 \%$ had clinical cure and they had a lower post-op ODI (7.1) in spite of comparable weight loss. Even though our patients had similar pre-op BMI but the mean pre-op ODI was less than most reported studies the reason for which is not clear but might be responsible for a much higher cure rate.

\section{REFERENCE}

1 Greenburg DL, Lettieri CJ, Eliasson AH. Effects of surgical weight loss on measures of obstructive sleep apnea: a meta-analysis. Am J Med 2009;122:535-42

\section{P117 COMPARISON OF THE EFFECTS OF CONTINUOUS POSITIVE AIRWAY PRESSURE AND MANDIBULAR ADVANCEMENT DEVICES ON SUBJECTIVE DAYTIME SLEEPINESS IN PATIENTS WITH OBSTRUCTIVE SLEEP APNEA: A NETWORK META-ANALYSIS}

DJ Bratton, T Gaisl, C Schlatzer, M Kohler. Department of Pulmonology, University Hospital Zurich, Zurich, Switzerland

\subsection{6/thoraxjnl-2015-207770.254}

Background Obstructive sleep apnoea (OSA) is associated with increased daytime sleepiness. Previous meta-analyses have shown that both continuous positive airway pressure (CPAP) and mandibular advancement devices (MADs) reduce the Epworth Sleepiness Score (ESS), a common measure of daytime sleep propensity. However, no meta-analysis has yet identified which treatment is superior in reducing ESS, perhaps due to a lack of studies directly investigating the two treatments. In addition, the effect of CPAP usage on ESS has yet to be thoroughly explored. Methods We searched Medline and the Cochrane Library up to the end of May 2015 to identify randomised controlled trials in OSA investigating the effect of CPAP and/or MADs against each other or an inactive control (IC, placebo or no treatment) on ESS. A network meta-analysis was used to incorporate both 